\title{
Efficient Cyclostationary Detection Based Spectrum Sensing in Cognitive Radio Networks
}

\author{
P. Venkatramana ${ }^{\# 1}$, S. Narayana Reddy ${ }^{\# 2}$ \\ \#1 Department of ECE, Sree Vidyanikethan Engineering College \\ ${ }^{\# 2}$ Department of ECE, $S$ V University College of Engineering
}

\begin{abstract}
As the customers in wireless communication becoming crowdy and it becomes an important to tackle the spectrum scarcity problem. Most of TV licensed spectrum band, users only utilize their chosen resources partially, thus force the need of cognitive radios (CR) which offers the capable feature of accessing the unused spectrum by dynamic spectrum.

In this paper, we are presenting the cyclostationary detection method for estimation and spectral autocorrelation function technique to analyze the spectrum. We used cyclostationary feature detection under modulation scheme to detect the primary users at very low SNR and enhancing cyclostationary feature detection with peak detection algorithm for effective performance. To reduce the noise peaks in the cyclostationary output absolute threshold, standard deviation and filtfilt are the techniques used to get a better efficiency for signal detection.
\end{abstract}

Keywords- Cyclostationary, Correlation Function, autocorrelation functions, cyclic frequency, Spectral Coherence.

\section{INTRODUCTION}

The idea of cognitive radio was first presented officially in an article by Joseph Mitola III and Gerald Q. Maguire, Jr in 1999. Different communication systems such as medical, broadband mobile telecommunication, marine communication, defense and emergency services utilize the radio spectrum. A report by the FCC

Spectrum Policy Task Force in November 2002 has stated that the spectrum scarcity problem is mostly due to the insufficient utilization of the RF spectrum rather than the lack of unoccupied frequency bands. With the growth of communication users, makes the spectrum more congested even though federal communication commission (FCC) has expanded some unlicensed spectrum bands for users. The overcrowded spectrum reduces overall quality of service for users in that allotment. To overcome spectrum scarcity a potential solution to this problem is COGNITIVE RADIOS. "Cognitive radio: A radio or system that senses its operational electromagnetic environment and can dynamically and autonomously adjust its radio operating parameters to modify system operation, such as maximize throughput, mitigate interference, facilitate interoperability, access secondary markets. [1]. Cognitive radio is one of the modern techniques for wireless communication systems to utilize the unused spread spectrum effectively. The motivation for Cognitive radio is a concept of utilizing licensed spectrum in an unlicensed manner without causing interference.

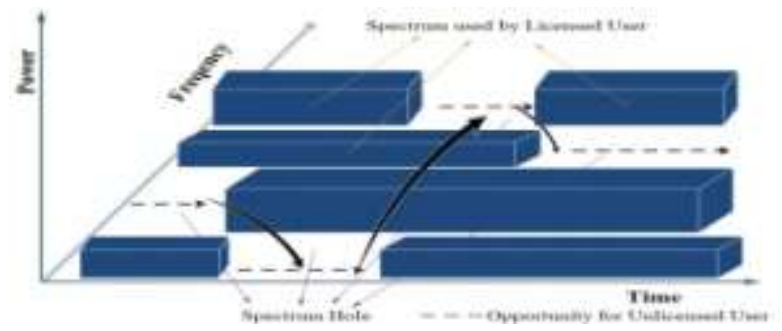

Fig 1: Representation of spectrum holes

To implement without Interference to the primary signal, the cognitive radio needs to sense the availability of the spectrum before accessing the channel [2]. So the ability of sensing an idle spectrum and the ability to temporarily utilize a spectrum without interfering with Primary Users are two essential components required for the success of cognitive radios [3]. In cognitive radio terminology, PU can be defined as the user who has license to use a specific part of the spectrum. On the other hand, secondary users (SU) do have license to use the spectrum but only when PU is absent.

Cognitive radio can sense the available spectrum for the secondary users when primary user is not using the allotted frequency spectrum, so that spectrum utilization can be improved. Cognitive radios are fully programmable wireless devices that can sense their environment and dynamically adapt their transmission waveform, channel access method, spectrum use, and networking protocols as needed for good network and application performance. Consequently, spectrum sensing performed by CR cannot be restricted to simply monitor the power in some frequency bands of interest but must include detection and identification in order to avoid interference. The main challenges with cognitive radios are that it should not interfere with the licensed users and should vacate the band when required. For this it should sense the signals faster. CR's are regarded as Transceivers that automatically detect (sense the existence of) available channels in a wireless spectrum and accordingly, change their transmission or reception parameters [3, 4]. This work focuses on the spectrum sensing techniques that are 
based on primary transmitter detection. Spectrum sensing plays an important role in cognitive radio (CR) systems; the fundamental problem of spectrum sensing is to discriminate an observation that contains only noise from an observation that contains a very weak signal embedded in noise. It is difficult to find vacant bands to deploy new services and enhance existing ones. To overcome this situation, we need an improved utilization of the spectrum which will create opportunities for Dynamic Spectrum Access (DSA).

An important aspect of a cognitive radio is spectrum sensing, which involves two main tasks: signal detection and modulation classification. Signal detection refers to detection of unused spectrum [4] which are called spectrum holes and it is shown in the Fig 1. This task is important so that the unlicensed users do not cause interference to licensed users. Modulation classification consists of automatically identifying the modulation scheme (PSK, FM, QPSK, etc) of a given communication system.

Spectrum sensing and estimation is the first step to implement Cognitive Radio system. Spectrum Sensing Methods are classified as Energy Detection, Matched filter detection and Cyclostationary Based Spectrum Sensing detection. Energy detection as a non coherent method is easy to implement but it cannot discriminate between the primary signal and noise, and hence makes it difficult to set the threshold used for primary user detection, especially at low SNR. Matched filter is an optimal detection technique but requires a prior knowledge of primary user signal for the detection. Cyclostationary feature detection can discriminate between the primary signal and noise, and no need of prior knowledge of primary user for spectrum sensing and produces efficient output even at low SNR Cases. Cyclostationary detector is based on the spectral redundancy present in almost every manmade signal. It is called a cyclic feature detector. The second order cyclostationary is used to extract sine-wave from the signal is introduced by Gardner in [5-6]. The mathematical functions used to characterize cyclostationary signals are Cyclic Autocorrelation Function (CAF) and Cyclic Domain Profile (CDP). Cyclic Domain Profile refers to the cyclic repetition of frequency [7]. In a cognitive radio system A primary system operated in the licensed band has the highest priority to use that frequency band (e.g. 3G/4G cellular, digital TV broadcast). Other unlicensed users/systems can neither interfere with the primary system in an Intolerable way nor occupy the license band [13].

\section{CYCLOSTATIONARY DETECTION}

In Cyclostationary signals, the mean value and autocorrelation function have periodicity. In this paper a signal is taken which can be called as primary signal

$$
X(t)=s(t)+w(t)
$$

Where $x(t)$ is the input transmitted signal $\mathrm{w}(\mathrm{t})$ is the noise signal (AWGN) and $\mathrm{s}(\mathrm{t})$ is the primary user signal

Cyclic spectral analysis deals with second order transformations of a function and its spectral representation. A function $x(t)$ is said to exhibit second order periodicity if spectral components of $\mathrm{x}(\mathrm{t})$ exhibit temporal correlation.

\section{A. Temporal Redundancy:}

A wide-sense cyclostationary signal $\mathrm{x}(\mathrm{t})$ exhibits a periodic autocorrelation function $[6,8]$. It has periodic components that can be found by $\mathrm{CR}$ to eliminate it from noise. A cyclostationary process is a signal having statistical properties that vary cyclically with time. A cyclostationary process can be viewed as multiple interleaved stationary processes. These processes are not periodic function of time but their statistical features indicate periodicities. The following conditions are essential to be filled by a process for it to be wide sense cyclostationary. The periodicity of the mean and autocorrelation functions are expressed by the equations are as follows:

$$
R_{x}(t, \tau)=E\left[x(t) x^{*}(t-\tau)\right]
$$

Mean function is expressed as

$$
m_{a}=E\{x(t)\}=0
$$

Since autocorrelation function is periodic it can be expressed by applying Fourier series which is decomposed as

$$
R_{x}(t, \tau)=\sum_{\alpha} R_{x}^{\alpha}(\tau) e^{j 2 \pi \alpha t}
$$

Where the sum is over integer multiples of the fundamental frequencies. The coefficient $\boldsymbol{R}_{x}^{\alpha}(\tau)$ is called the cyclic autocorrelation function, and represents the Fourier coefficient of the series given by

$$
R_{x}^{\alpha}(\tau)=\frac{1}{T_{0}} \int_{t=-T_{0} / 2}^{T_{0} / 2} R_{x}(t, \tau) e^{-j 2 \pi \alpha t} d t
$$

The autocorrelation function is replaced by its time average which is represented as

$$
R_{x}^{\alpha}(\tau)=\lim _{T \rightarrow \infty} \frac{1}{T} \int_{t=-T_{0} / 2}^{T_{0} / 2} x(t) x^{*}(t-\tau) e^{-j 2 \pi \alpha t} d t
$$

The cyclic autocorrelation is therefore intuitively obtained by extracting the frequency $\alpha$ sine- wave from the time-delay product $\mathrm{x}(\mathrm{t}) \mathrm{x} *(\mathrm{t}-\tau)$. The Spectral correlation density (SCD) $s_{x}^{\alpha}(f)$ is defined as the Fourier transform of $R_{x}^{\alpha}(\tau)$ over $\tau$.

\section{B. Spectral Redundancy:}

The Fourier transform of $x(t)$ is $X(f)$. The SCD measures the degree of spectral redundancy between the frequencies $f-\alpha / 2$ and $f+\alpha / 2$ ( $\alpha$ is the cyclic frequency). The Fourier transform of autocorrelation function is defined as Spectral Correlation Function (SCF) [12] and is expressed as

$$
s_{x}^{\alpha}(f)=\int_{-\infty}^{\infty} R_{x}^{\alpha}(\tau) e^{-j 2 \pi f t} d \tau
$$

It can be mathematically expressed as the correlation between two frequency bins centered on $f-\alpha / 2$ and $f+\alpha / 2$ when their width tends toward zero $[6,8]$.

$$
S_{x}^{\alpha}(f)=\lim _{T \rightarrow \infty} \lim _{\Delta t \rightarrow \infty} \frac{1}{T \Delta t} \int_{t=-\Delta t / 2}^{\Delta t / 2} X_{T}\left(t, \frac{f+\alpha}{2}\right) X_{T}^{*}\left(t, \frac{f-\alpha}{2}\right) d t
$$


In practice there are only a limited number of samples available and hence SCF needs to be estimated from these samples. Let us define the cyclic periodogram as [9][10].

$$
S_{x T}^{\alpha}(t, f)=\frac{1}{T} X_{T}\left(t, \frac{f-\alpha}{2}\right) X_{T}^{*}\left(t, \frac{f-\alpha}{2}\right)
$$

Where $\mathrm{X}_{\mathrm{T}}(\mathrm{t}, \mathrm{f})$ is the short-time Fourier transform of signal

$$
X_{T}(t, f)=\int_{t-T / 2}^{t+T / 2} x(u) e^{-j 2 \pi f u} d u
$$

SCF can be obtained by increasing the observation length $\mathrm{T}$ and decreasing $\Delta \mathrm{t}$.

$$
s_{x}^{\alpha}(f)=\lim _{T \rightarrow \infty} \lim _{\Delta t \rightarrow 0} S_{x T}^{\alpha}(t, f)
$$

\section{Spectral Coherence and $\alpha$-Profile:}

SCF is a correlation of frequency components shifted by $f+\frac{\alpha}{2}$ and $f-\frac{\alpha}{2}$. It is intuitive to define Spectral Coherence (SC) [11] as

$$
C_{x}^{\alpha}=\frac{S_{x}^{\alpha}(f)}{\left[S\left(f+\frac{\alpha}{2}\right) S\left(f-\frac{\alpha}{2}\right)\right]^{1 / 2}}
$$

The magnitude of SC is always between 0 and 1 . In order to reduce the computational complexity, one just uses the Cyclic Domain Profile (CDP) or $\alpha$-profile which is defined as

$$
I(\alpha)=\max _{f}\left|C_{x}^{\alpha}(f)\right|
$$

The process is shown in the Fig. 2 for better understanding.

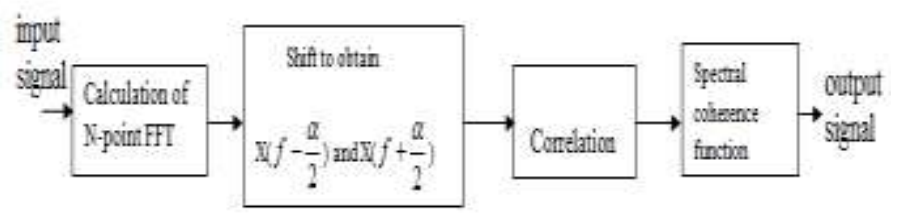

Fig 2: Extraction of spectral coherence function

\section{Signal detection}

All man-made signals and modulated signals exhibit second order cyclostationary. From the CDP of the signal, important information about the signal like modulation type, keying rate, pulse shape, and carrier frequency can be obtained, [12]. Fig. 4 shows the CDP for QPSK. When SCF is plotted, the occupancy status of the spectrum can be found out. If a primary user signal is present in the operating frequency range, the SCF gives a peak at its centre. The peak will not be present in the case when there is no primary user signal present in the concerned frequency range. Spectrum sensing is used to determine the presence or absence of primary users so we need to distinguish between these two hypotheses [14];

First we need to determine the threshold $\mathrm{C}_{\mathrm{TH}}$. For signal detection and when no signal is present, i.e. $x(t)=n(t), C T H$ will use the relationship as [15];

$$
C_{T H}=\max \left[I(\alpha) / \sqrt{\left.\left(\sum_{\alpha=0}^{N} I^{2}(\alpha)\right) / N\right]}\right.
$$

$\mathrm{N}$ is the length of observation data

We can distinguish signal from noise by analyzing the SCD function. Furthermore, it is possible to distinguish the signal type because different signals may have different nonzero cyclic frequencies. Cyclostationary detection block contains a FFT, AWGN, correlate, average over threshold and a feature detection block is shown in the Fig.3.

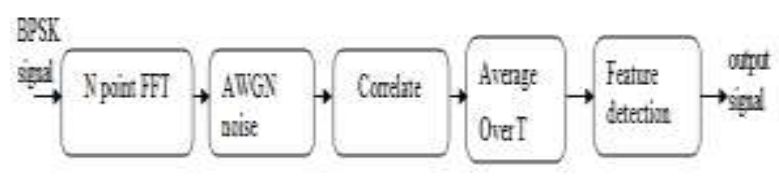

Fig 3: cyclostationary detection

A random discrete signal is taken and modulated using different modulation schemes. The CFD basically contains filters, ADC, quantizer, encoder, and fft blocks. In this paper we use fast Fourier transform (FFT) and a Noise is added by AWGN block. Cyclostationary feature detection method deals with the inherent cyclostationary properties or features of the signal. Such features have a periodic statistics and spectral correlation that cannot be found in any interference signal or stationary noise. It exploits this periodicity in the received primary signal to identify the presence of primary users, and that is why the cyclostationary feature detection method possesses higher noise immunity than any other spectrum sensing method. The output is taken using spectrum analyzer which displays the output in a graphical form which can be easily understandable. The output plot thus obtained is the cyclic SCF. Peak detection algorithm is used for the Cyclostationary output. The plot between probability of detection and SNR is termed as the receiver operating characteristics; using sensing algorithm the cyclostationary detection method, shows that the primary signal is present, and probability of detection increases with Different SNR values.

\section{TECHNIQUES APPLIED}

The Cyclic Domain Profile for QPSK Signals are extracted and for the obtained signals the following techniques are applied to minimize the noise for better probability of detection. Fig shows the Cyclic Domain Profile of QPSK signal.

$$
\begin{aligned}
& H_{0}: x(t)=n(t) \\
& H_{1}: x(t)=s(t)+n(t)
\end{aligned}
$$




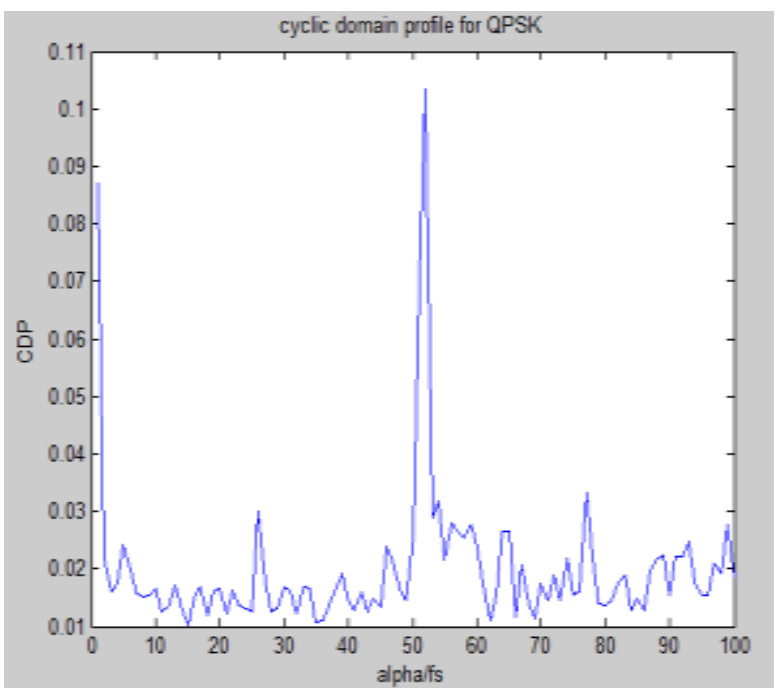

Fig 4: cyclic domain profile for QPSK signal

\section{Obsolute Threshold:}

In some applications we do not need to know the exact peak amplitudes and locations, rather we need to know the number or general location of peaks, in this case we use an absolute threshold function. The absolute threshold can be influenced by several different factors such as motivations, expectations, and cognitive processes, that whether the subject is adapted to the stimulus. The absolute threshold can be compared to the difference threshold which is the measure of how two different stimuli must be for the subject to notice that they are not the same. For the cyclostationary output the absolute threshold is applied where it reduces the noise peaks to a minimum. Fig 5 shows reduction of noise peaks at different frequencies, and a centre peak that indicates the probability of detection. The noise peaks are diminished by considering an absolute value of Cyclostationary output.

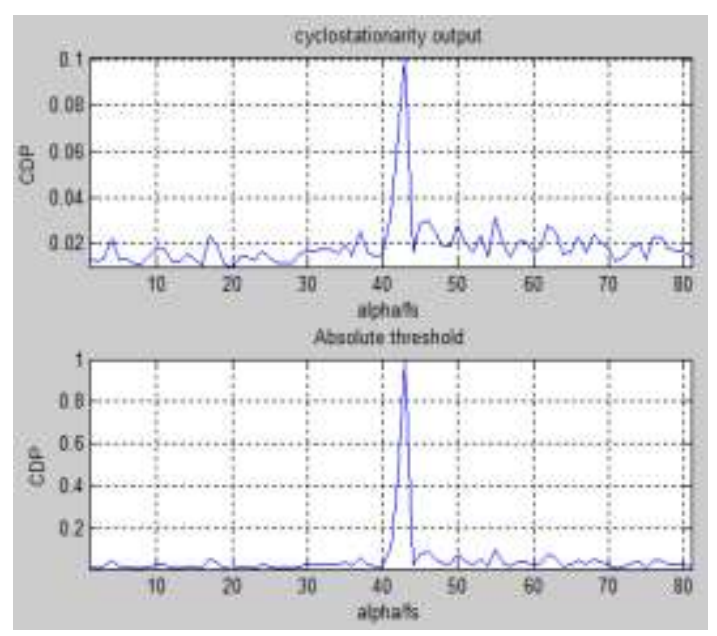

Fig 5: Detection of peak signal using absolute threshold

\section{Standard deviation:}

Standard deviation is a measure for how much the frequencies in a spectrum can deviate from the centre of gravity. For a sine wave the Standard deviation is zero and hence by increasing the number of samples the noise peaks are diminished as shown in the Fig 6. The standard deviation diminishes the noise peaks more than the absolute threshold based on the increased number of samples.

$$
\begin{gathered}
S=\left[\frac{1}{n-1} \sum_{i=1}^{n}\left(x_{i}-\bar{x}\right)^{2}\right]^{1 / 2} \\
\text { Where } \quad \bar{x}=\left[\frac{1}{n} \sum_{i=1}^{n} x_{i}\right]
\end{gathered}
$$

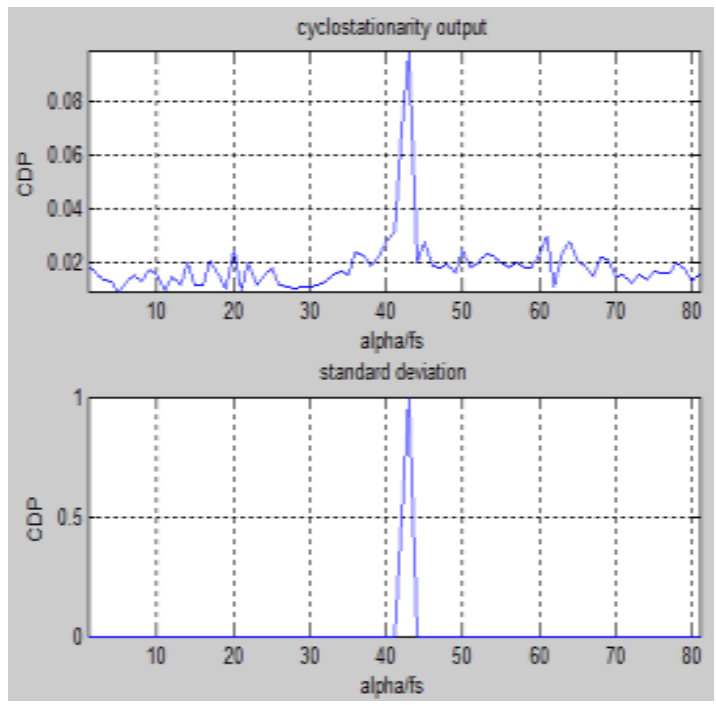

Fig 6: Detection of peak signal using standard deviation

\section{Filtfilt:}

Filtfilt is a zero phase forward and Reverse filtering, after filtering in the forward direction; the filtered sequence is then reversed and run back through the filter. The Filter is described by the difference equation:

$$
\begin{aligned}
& y(n)=b(1) * x(n)+b(2) * x(n-1)+\ldots+b(n b+1) * x(n-n b)- \\
& a(2) * y(n-1)-\ldots-a(n a+1) * y(n-n a) .
\end{aligned}
$$

Where $\mathrm{y}$ is the time reverse of the output of the second filtering operation. The result has precisely zero phase distortion and magnitude modified by the square of the filter's magnitude response. The length of the input $x$ must be more than three times the filter order, defined as $\max ($ length(b)-1, length(a)-1). FILTFILT should not be used with differentiator and Hilbert FIR filters, since the operation of these filters depends heavily on their phase response. Fig 7 shows the output of the filtfilt command where the noise is completely diminished by using the difference equation. By comparing with the other two techniques filtfilt is the best method for reducing the noise by filtering. 


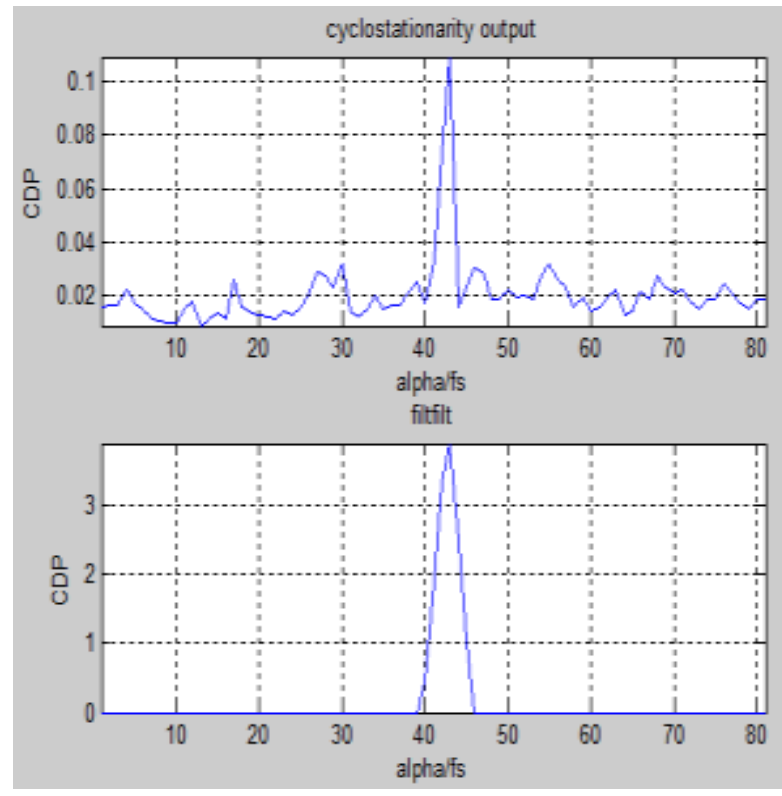

Fig 7: Detection of peak signal using filtfilt technique

Finally for the obtained signal the Probability of detection for different SNR is calculated and plotted which is shown in Fig 8.

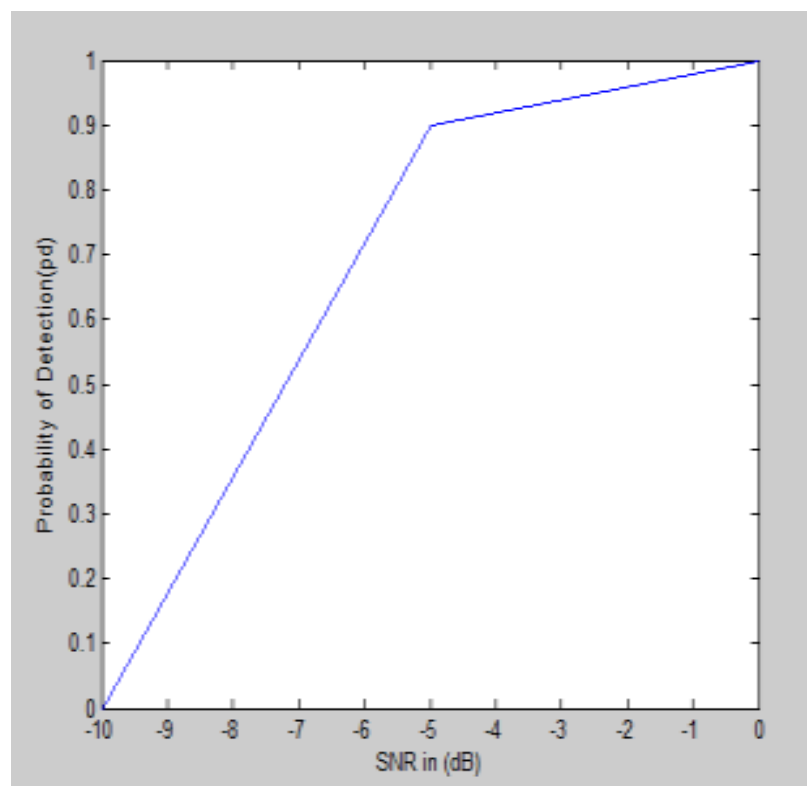

Fig 8: Probability of detection for different SNR.

\section{CONCLUSION}

In this paper, we have presented the peak detection algorithm for estimation and detection of the primary signal by applying certain threshold and techniques to analyze the spectrum. if the peak signal is present at the centre of the SCF then it is said to be that primary user is present if not the primary user is absent and the secondary user can occupy the spectrum band and if primary user needs the spectrum band the secondary user must vacate the spectrum by sensing the spectrum using spectrum sensing technique. Among the techniques the filtfilt outperforms. Thus the Cyclostationary detection is best suited for very low SNR.

\section{REFERENCES:}

[1] Sajjad Ahmed Ghauri, Ijaz Mansoor Qureshi, Ihtesham Shah and Nasir Khan, " Modulation Classification using Cyclostationary Features on Fading Channels, "Research Journal of Applied Sciences, Engineering and Technology 7(24): 5331-5339, 2014.

[2] J.Mitola, -Cognitive radio: making software radio more personal, \| IEEE Personal Communications, vol. 6, no. 4, pp. 13-18, 1999.

[3] Samuel Cheng, 2012, Foundation of Cognitive Radio Systems,

[4] S. Haykin. Cognitive radio: brain-empowered wireless communications. Selected Areas in Communications, IEEE Journal on, 23(2):201\{220, 2005.

[5] I. F. Akyildiz, L. Won-Yeol, and K.R. Chowdhury., " Spectrum management in cognitive radio adhoc networks." IEEE Xplore, $3(12): 6\{12,2009$

[6] W. A. Gardner, Statistical Spectral Analysis: A Nonprobabilistic Theory, Prentice-Hall, Englewood Cliffs, NJ, USA, 1987.

[7] W. A. Gardner, "Signal interception: a unifying theoretical framework for feature detection," IEEE Transactions on Communications, vol. 36 , no. 8, pp. 897-906, 1988 .

[8] FCC, "Spectrum Policy Task Force Report," vol. ET Docket No. 02-155, November 2002.

[9] W. A. Gardner and C. M. Spooner, "Signal interception: performance advantages of cyclic-feature detectors," IEEE Transactions on Communications, vol. 40, no. 1, pp. 149-159, 1992

[10] R. S. Roberts, "Computationally efficient algorithms for cyclic spectra analysis," IEEE Signal Processing Magazine, Apr. 1991.

[11] W. M. Gardner, "Measurement of spectral correlation," IEEE Trans on Acoust, Speech, and Signal Processing, vol. ASSP-34, no. 5, Oct.1986.

[12] Barathram Ramkumar, Tamal Bose, Miloje S. Radenkovic, "Combined Blind Equalization and Automatic Modulation Classification for Cognitive Radios".

[13] Julien Renard, Jonathan Verlant-Chenet, Jean-Michel Dricot, Philippe De Doncker, and Francois Horlin, "Higher-Order Cyclostationarity Detection for Spectrum Sensing".EURASIP Journal onWireless Communications and Networking Volume 2010.

[14] niyati Sohni, anand Mane, "Spectrum Sensing by Cyclostationary Detector," International Journal of Industrial Electronics and Electrical Engineering, ISSN: 2347-6982 Volume-2, Issue-7, July2014.

[15] Kyouwoong Kim, I. A. Akbar, K. K. Bae, Jung-sun Urn, C. M. Spooner, 1. H. Reed, "Cyclostationary approaches to signal detection and classification in cognitive radio", 2nd IEEE International Symposium on New Frontiers in Dynamic Spectrum Access Networks, pp.212-215, (2007).

[16] W. Gardner, "The spectral correlation theory of cyclostationary timeseries", Signal Processing, Vol. 11(1) pp.13-36, (1986). 


\section{AUTHORS}

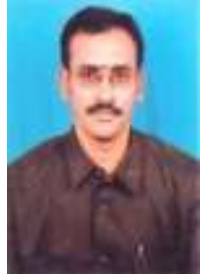

Mr. P. Venkat Ramana received his B.Tech from Madras University, Chennai and M.Tech from Sri Venkateswara University, Tirupati. He is currently Professor in the Department of Electronics and Communication Engineering at Sree Vidyanikethan Engineering College, Tirupati and working towards Ph.D. in the Department of Electronics and Communication Engineering, Sri Venkateswara University College of Engineering, Tirupati.

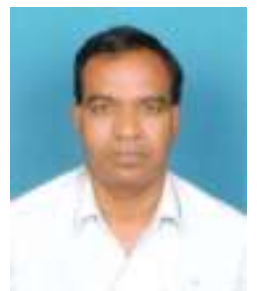

Dr. S. Narayana Reddy worked as Scientist in SAMEER in the design of MST RADAR system for 4 years and later joined as Assistant Professor in the Department of EEE at S.V. University Tirupati, INDIA. He has 25 years of experience in teaching and research. Presently he is working as Professor in the department of ECE at S.V.University. He is life Member of ISTE, fellow of IETE, fellow IE(I). He has published more than 80 papers in various National and International papers/conferences and guided 9 Ph.D scholars, His current interests include radar systems, signal processing and wireless communication systems. 